www.revistad y o.com

\title{
Crisis económicas y su influencia en la siniestralidad laboral
}

\author{
Sonia Contreras Ovejero, Miguel Ángel Manzanedo del Campo, Álvaro Herrero Cosío.
}

Recibido: 24 de Enero de 2018 / Aceptado: 15 de Febrero de 2018

\section{Resumen}

Los distintos tipos de crisis cambian el desarrollo de los sucesos de forma brusca, generando situaciones distintas que pueden afectar al ser humano modificando sus comportamientos. Por ello, se pretenden analizar estos nuevos escenarios para poder hacer predicciones que permitan paliar, de alguna manera, sus efectos así como sus consecuencias.

Las crisis económicas han afectado al empleo, lo que puede haber generado una modificación de las condiciones de trabajo. El mantenimiento de un mejor nivel de seguridad y salud entre los trabajadores es un objetivo social fundamental ya que los accidentes laborales, los trastornos psicológicos o las enfermedades profesionales generan un menor bienestar personal y social.

En este trabajo se pretende analizar el comportamiento de las variables macroeconómicas durante los periodos de crisis y también la evolución de la siniestralidad laboral durante estos mismos períodos, al objeto de aportar información que permita tomar decisiones directivas para mejorar las condiciones de seguridad y salud laboral. Con este objetivo se aplican, de manera novedosa, redes neuronales artificiales para el análisis de distintos datos.

\section{Palabras clave}

Seguridad y Salud Laboral, Accidentes de Trabajo, Siniestralidad Laboral, Variables Macroeconómicas, Crisis Económicas, Redes Neuronales Artificiales.

\section{Introducción}

Las economías de los diferentes países experimentan el paso por diferentes estados a lo largo del tiempo; así, a un estado de crecimiento económico le sigue una situación de decrecimiento económico, configurando distintos ciclos económicos. Entre uno y otro estado, la economía atraviesa por lo que se conocen como épocas o periodos de transición.
En momentos de crisis, los Indicadores Macroeconómicos sufren, por lo general, una evolución adversa, decrece el PIB, aumenta la inflación (IPC), aumenta el desempleo, crece la deuda pública, aumenta la prima de riesgo, crece la morosidad bancaria, etc. En el gráfico 1 se muestran los distintos ciclos económicos por los que ha atravesado la economía española a lo largo de los últimos veinticinco años, período temporal seleccionado para este estudio.

\section{Gráfico 1 Ciclos Económicos en España \\ Fuente: Elaboración propia}

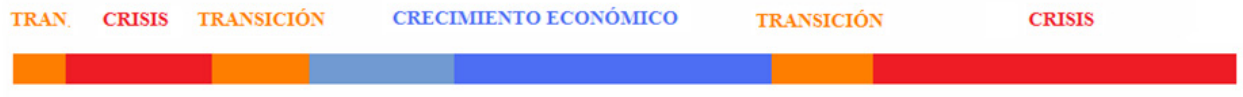

\begin{tabular}{|l|l|l|l|l|l|l|l|l|l|l|l|l|l|l|l|l|l|l|l|l|l|l|l|l|}
1990 & 1991 & 1992 & 1993 & 1994 & 1995 & 1996 & 1997 & 1998 & 1999 & 2000 & 2001 & 2002 & 2003 & 2004 & 2005 & 2006 & 2007 & 2008 & 2009 & 2010 & 2011 & 2012 & 2013 & 2014 \\
\hline
\end{tabular}
El presente trabajo se desarrolla en el ámbito geográfico del estado español, en el período comprendido entre los años 1990 a 2014, en el que se registraron dos crisis económicas a nivel nacional: La crisis de los años noventa (de 1992 a 1994) y la actual crisis (iniciada a mediados del año 2007).

Respecto a la primera, en el trabajo desarrollado por Vázquez (2007) se muestra como desde 1992 se inicia una época de recesión en nuestro país, dando lugar a un incre-

Miguel Ángel Manzanedo del Campo -

Universidad de Burgos mmanz@ubu.es

Álvaro Herrero Cosío - Universidad de Burgos ahcosio@ubu.es mento muy notable del desempleo, una caída inusitada de los beneficios y de la inversión de las empresas, un volumen de deuda pública muy elevado y un déficit del conjunto de las Administraciones Públicas superior al 7\% del Producto Interior Bruto (PIB). Posibles causas de dicha crisis podrían haber sido, la elevación del precio de las materias primas y del petróleo, la desmonetización del oro y la flotación libre de las monedas. Las cuales provocaron una elevación de los costes de producción, la aparición del déficit y por último, una espiral inflacionista de precios y salarios, que como consecuencia generaron un aumento del nivel del paro.

En cuanto a la actual crisis, se observan numerosas interpretaciones tanto a nivel mundial como a nivel nacional (Jimeno y Santos, 2014). Es en marzo de 2007 aproximadamente, cuando la actual crisis tiene su origen en España. En agosto del mismo año, hubo un desmoronamiento de los 
activos hipotecarios que afectó de forma directa a muchas entidades bancarias de Europa y de los EEUU, provocando un choque directo sobre los mercados financieros, y es en octubre de ese mismo año cuando aparecen las hipotecas subprime cuyas repercusiones comenzaron a manifestarse de manera grave al inicio del año 2008.

Según algunos expertos, la situación económica mundial junto con otros factores, producen el estallido de la burbuja inmobiliaria española, la cual parece ser el detonante definitivo de esta crisis en nuestro país. Aunque también hay detractores del hecho de que el sector de la construcción fuera el único detonante de esta crisis, pues Alonso-Nuez et al. (2015), señalan en su investigación que "la importancia atribuida a este sector no se corresponde con su verdadero papel en la economía española en general”.

Respecto a la siniestralidad laboral, indicar que desde el siglo pasado ha ido aumentando el interés sobre la misma, principalmente en los países más avanzados del mundo. Esta problemática supone un perjuicio, un deterioro y un desgaste importante en la víctima del accidente, y a su vez, conlleva un quebranto social en el entorno del accidentado y una pérdida económica para la empresa y para la víctima del accidente, debido a la ausencia o imposibilidad de ejercer su actividad laboral (Papazoglou et al., 2015).

En España se observan altos niveles de siniestralidad laboral ya que cada día mueren del orden de tres personas por accidente laboral, estimándose un gasto en torno al 1\% de PIB (INE 2014); mientras que en países como Suecia o Gran Bretaña (Eurostat, 2013), sus cifras son entre cuatro y cinco veces menor que en España. Por ello, surge la necesidad y el interés de profundizar en el conocimiento de la siniestralidad laboral en nuestro país.
Un estudio de costes de accidentes de trabajo fue realizado por Manzanedo y Saiz (1996), donde se analizan todos los costes derivados de cada accidente. Así mismo se elabora un modelo de cálculo de costes de accidentes, relacionando los costes asegurados y no asegurados. También García y Montuenga (2009) investigan la influencia de los trabajadores y de los puestos de trabajo que ocupan, en la incidencia de accidentes laborales según la gravedad de éste, coligiendo que los trabajadores con mayor experiencia laboral presentan menos riesgo de tener un accidente grave o mortal; añadiendo, que para reducir la incidencia de los accidentes mortales habría que limitar el número de horas trabajadas, especialmente en los casos en que no se cuente con una amplia experiencia laboral. Algunos otros expertos reclaman la promoción y el apoyo de la prevención como herramienta para la reducción de la siniestralidad laboral, así como la implementación de una cultura y prácticas laborales que proporcionen un ambiente capaz de promover la efectividad de la organización, más aún en épocas difíciles como la actual, lo que provocará un ahorro económico a largo plazo (Teixidó, 2009).

Son escasas las investigaciones publicadas sobre el estudio conjunto de crisis económicas y siniestralidad laboral. Así, Moënne (2008) afirma que en tiempos de bonanza económica aumenta el estrés laboral y disminuye el tiempo disponible para la prevención, lo cual termina afectando a la siniestralidad laboral. También (Villanueva y Clemente, 2001) analizan la variación de la siniestralidad en relación con los cambios del ciclo económico, exponiendo que la siniestralidad sufre un descenso en fases de recesión, mientras que en fases expansivas de la economía, se produce un incremento del empleo con contratos precarios y con mayor riesgo de accidente, como puede observarse en el gráfico 2 .
Gráfico 2 Accidentes Laborales con Baja en Jornada de Trabajo en España (sin In Itinere, sin Recaídas)

Fuente: Elaboración propia con datos del MEYSS

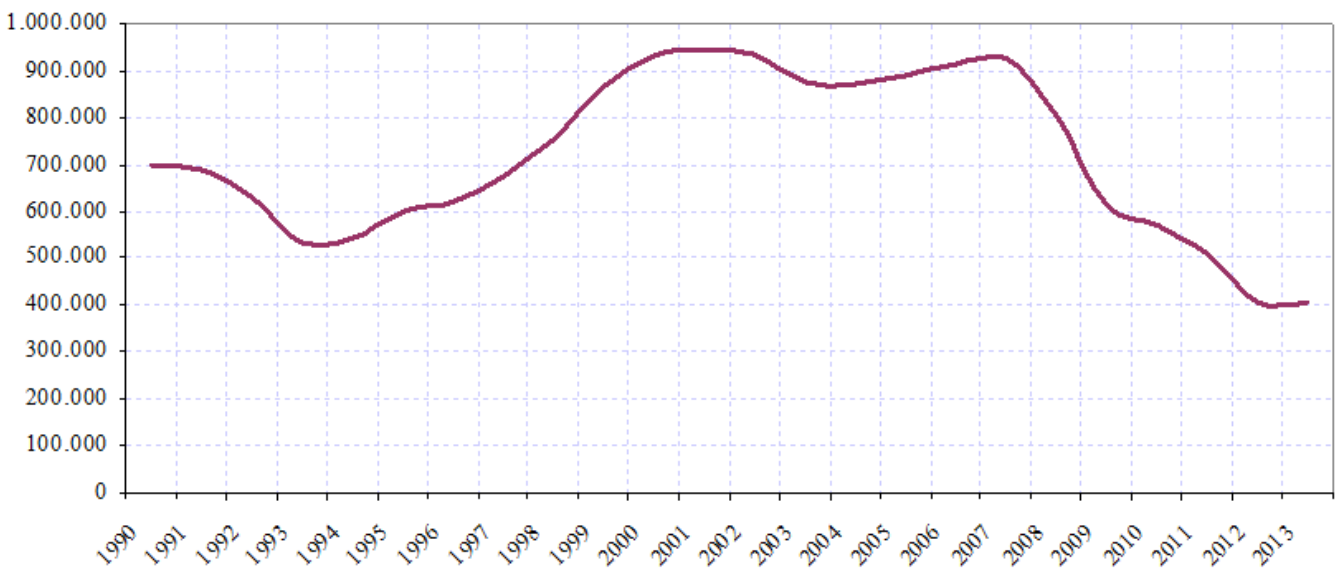


Autores como (Alcazar, 2014), analizan el legado que nos deja esta crisis tras los duros ajustes económicos realizados en nuestro país, deduciendo que sus efectos se observarán durante años en la economía, afectando al PIB, la inflación, los salarios, el sueldo, las empresas, la financiación, el consumo, etc.

La siniestralidad laboral en España ha podido ser influida por las crisis económicas de los 90 y la actual, siendo necesario conocer la relación que pueda existir entre distintos indicadores representativos de ambos conceptos. Por ello este trabajo pretende profundizar en la relación entre crisis económica y siniestralidad laboral, para conocer la posibilidad de considerar a la crisis económica como una variable relacionada con la siniestralidad laboral y, así, aportar nuevos conocimientos que permitan mejorar las condiciones de seguridad y salud.

\section{Desarrollo experimental}

A continuación se expone el diseño experimental realizado en el presente trabajo, fundamentalmente en lo relacionado con los datos analizados, así como los métodos empleados en dicho análisis.

\subsection{Datos}

Para el estudio planteado se han empleado datos procedentes del Instituto Nacional de Seguridad e Higiene en el Trabajo del Gobierno de España (INSHT), del Instituto Nacional de Estadística del Gobierno de España (INE), del Banco de España (BDE) y del Ministerio de Empleo y Seguridad Social del Gobierno de España (MEYSS).

Han sido empleados los valores de las series anuales de algunas de las variables macroeconómicas más relevantes y, también, las bases de datos oficiales sobre la accidentalidad laboral, junto con las bases de datos oficiales del número de afiliados a la Seguridad Social. Además, se han utilizado los índices de incidencia registrados en la página web oficial del MEYSS.

El número total de accidentes analizados en el presente trabajo asciende a diecisiete millones doscientos veintiún mil setenta (17.221.070). Además, cabe destacar que este número no incluye los accidentes in itínere, los accidentes que no ocasionaron ningún día de falta al trabajo, ni las recaídas producidas por los mismos. Estos datos junto con otros como sexo, edad, gravedad, incidencia, etc., se examinan utilizando distintas variables de siniestralidad que nos pueden aportar información de alta relevancia sobre el accidentado y el accidente.

Las variables macroeconómicas se utilizan en todas las economías del mundo y nos permiten comparar distintos va- lores, tanto a nivel nacional (variación anual), como a nivel global (entre diferentes países). El estudio macroeconómico nos permitirá conocer el comportamiento de nuestra propia economía, y si afectan de algún modo o tienen alguna relación con aspectos como el sexo, la edad y el resto de variables de siniestralidad. Las variables macro utilizadas para este estudio son el Productor Interior Bruto (PIB), la Inflación (IPC), el Desempleo (número de parados), los Ocupados, los Asalariados, el Ahorro Nacional Bruto (ANB), la Remuneración de Asalariados, los Sueldos y Salarios Brutos (SSB), la Ganancia Media por Trabajador, el Salario Mínimo Interprofesional (SMI), las Jornadas No Trabajadas (JNT), los Trabajadores en Alta Laboral y el Coste Salarial por Trabajador, entre otras.

\subsection{Métodos}

Para el análisis de los datos multidimensionales, asociados tanto a las variables macroeconómicas como a las de siniestralidad por años, se han aplicado métodos como Principal Component Analysis (PCA), Cooperative Maximum Likelihood Hebian Learning (CMLHL) y Self-Organizing Map (SOM), que nos permiten representar de distintas maneras la estructura de datos multidimensionales de los que partimos.

Aunque son múltiples sus aplicaciones y muchos los investigadores que han trabajado con dichos métodos en diferentes áreas de conocimiento, consideramos que es la primera vez que se emplean en el estudio de crisis económicas y la siniestralidad laboral conjuntamente. Para ello, en primer lugar se ha realizado un estudio de las variables macroeconómicas por año con los métodos citados anteriormente. Después se ha llevado a cabo el mismo estudio pero en este caso para las variables de siniestralidad.

Al objeto de conocer sus características más significativas, a continuación se realiza una breve descripción individual los distintos métodos aplicados.

\subsubsection{Principal Component Analysis}

El Análisis de Componentes Principales (Principal Component Analysis - PCA) es un método estadístico basado en el análisis de información, mediante el reajuste de la dimensión (reducción del número de variables). Así, ante un conjunto de datos con múltiples variables, su finalidad es reducir la cantidad de éstas, tratando de minimizar la pérdida de información, en la medida de lo posible, para los nuevos datos. Los nuevos factores o componentes principales obtenidos con dicho reajuste, son el resultado de una combinación lineal de las variables originarias y, a su vez, serán independientes entre sí.

Esta técnica fue inicialmente desarrollada por Pearson (1901) a principios del siglo XX y posteriormente estudia- 
da y desarrollada por Hotelling (1933) en los años treinta. Con ésta técnica se consigue una doble función; por un lado, permite representar óptimamente en un espacio de reducida dimensionalidad las observaciones de un espacio general $\mathrm{N}$-dimensional (las componentes principales son el primer paso para identificar las posibles variables latentes, o no observadas que generan los datos) y por otro lado, permite transformar las variables originales, en general correlacionadas, en nuevas variables decorrelacionadas, facilitando la interpretación de los datos.

Según Hotelling (1933) y Pearson (1901), PCA es un método estadístico para la reducción de la dimensionalidad para conjuntos de datos de alta dimensionalidad. O lo que es lo mismo, si se parte de un conjunto de datos multivariable, la finalidad es reajustar este a un menor número de variables, en orden decreciente de importancia y con la mínima pérdida de información, de manera que las variables resultantes sean combinación lineal de las variables origen, e independientes entre sí. Cuando se proyectan los datos sobre los primeros componentes principales, éstos consiguen reducir la dimensionalidad que se verá acompañada de tanta varianza en los datos como sea posible.

Según Bishop (1995), PCA se puede describir como un mapeo de vectores $\mathrm{X}^{\mathrm{d}}$ en un espacio $\mathrm{N}$-dimensional en vectores $\mathrm{Y}^{\mathrm{d}}$ en un espacio $\mathrm{M}$-dimensional, siendo $\mathrm{M}<=\mathrm{N}$. Mientras que $\mathrm{X}$ puede ser representado como una combinación lineal de un conjunto de $\mathrm{N}$ vectores ortonormales $\mathrm{W}_{\mathrm{i}}$ :

$$
x=\sum_{i=1}^{N} y_{i} W_{i}
$$

Los vectores $\mathrm{W}_{\mathrm{i}}$ satisfacen la siguiente relación de ortonormalidad:

$$
W_{i}^{t} W_{j}=\delta_{i j}
$$

\section{Donde $\delta_{i j}$ es la delta de Kronecker.}

Este método de análisis (PCA) lo han empleado múltiples investigadores en sus estudios, algunos como Huang et al. (2015), Liu et al. (2014), Herrero et al. (2010), Wang et al. (2015) y Lozano (1999), entre muchos otros, pero el presente estudio es pionero en la aplicación de dicho método de análisis a la investigación sobre las crisis y la siniestralidad laboral.

\subsubsection{Cooperative Maximum Likelihood Hebian Learning}

Cooperative Maximum Likelihood Hebbian Learning (CMLHL), es un modelo de arquitectura neuronal no supervisada que se caracteriza por su capacidad para conservar un grado de orden global en los datos. Esta técnica permite un ordenamiento topológico de las distintas neuronas, atendiendo a una regla de vecindad o similitud, permitiendo llevar a cabo una búsqueda de proyecciones exploratorias (Exploratory Proyection Pursuit - EPP). EPP (Friedman y Tukey, 1974) se emplea para resolver problemas en los que es difícil identificar la estructura interna en datos de alta dimensionalidad. Para ello, se debe definir un índice que mida cómo es de interesante una proyección. Después, los datos se transforman maximizando dicho índice con el objetivo de maximizar el interés de acuerdo con él.

Una implementación neuronal de EPP es Maximum Likelihood Hebbian Learning (MLHL) (Corchado y Fyfe, 2003; Corchado et al., 2004). Las operaciones de este modelo neuronal se definen como:

Paso hacia delante:

$y_{i}=\sum_{j=1}^{N} W_{i j} x_{j}, \forall i$

Paso hacia atrás:

$e_{j}=x_{j}-\sum_{i=1}^{M} W_{i j} y_{i}$

Actualización de los pesos:

$\Delta W_{i j}=\eta y_{i} \operatorname{sign}\left(e_{j}\right)\left|e_{j}\right|^{p-1}$

Sobre MLHL se propone la inclusión de conexiones laterales (Corchado y Fyfe, 2003; Seung et al., 1998) que se derivan de la Distribución Gaussiana Rectificada (DGR) y se basan en distribuciones cooperativas (Seung et al., 1998), generando el modelo conocido como CMLHL. Este añade la aplicación de conexiones laterales entre las neuronas de la capa de salida, que se lleva a cabo entre el paso hacia atrás (o realimentación) y la actualización de pesos. Las conexiones laterales son aplicadas de acuerdo con la siguiente ecuación:

$y_{i}(t+1)=\left[y_{i}(t)+\tau(b-A y)\right]^{+}$

Donde es la rectificación necesaria para que los valores permanezcan en el cuadrante positivo y la fuerza de las conexiones laterales entre las neuronas de la capa de salida. Para que el algoritmo converja a un punto estacionario de la función de energía (generalmente un mínimo local), se debe escoger un valor adecuado para (Bertsekas, 1999).

Mediante la adición de las conexiones laterales, el modelo neuronal resultante (CMLHL) es capaz de identificar un cierto tipo de orden global o estructura en el conjunto de datos.

En el presente estudio, CMLHL ayuda a la identificación en la estructura asociada a los diferentes años del periodo de tiempo seleccionado, situando estos en la proyección obtenida por dicho modelo. En esta, se tiene en cuenta la similitud entre sí de los diferentes años, basándose en las variables macroeconómicas en primer lugar, en las de siniestralidad 
después, y por último, en ambos tipos de variables, de forma conjunta.

Son múltiples los expertos que han trabajan con este modelo neuronal previamente, en distintos campos de aplicación (Herrero et al., 2006; Herrero et al., 2010; Herrero et al., 2011; Sánchez et al., 2013), pero se aplica en el presente trabajo por primera vez en el estudio de la variación de los ciclos económicos (crisis económicas principalmente) y su relación con la siniestralidad laboral.

\subsubsection{Self-Organizing Map}

En 1982 Teuvo Kohonen presentó un modelo de red denominado como Self-Organizing Maps (SOM) o mapas auto-organizados, sobre la base de ciertas pruebas halladas a nivel cerebral. Es un tipo de red neuronal artificial con aprendizaje no supervisado, que obtiene una representación discreta en el espacio de las muestras de entrada, llamado mapa. Los mapas auto-organizados son diferentes de otras RNA, en el sentido que estos usan una función de proximidad (cercanía), con la intención de preservar las propiedades topológicas del espacio de entrada. Esto quiere decir que frente a datos similares en el espacio de entrada, responderán neuronas próximas entre sí, en el SOM. Se trata por lo tanto de un modelo neuronal que lleva a cabo un mapeo y no una proyección de los datos.

Gracias a la función de proximidad o vecindad en la que se fundamentan los SOM, este modelo debe descubrir rasgos comunes, regularidades, correlaciones o categorías en los datos de entrada, e incorporarlos a su estructura interna de conexiones. Se dice, por tanto, que las neuronas deben auto-organizarse en función de los estímulos (datos) procedentes del exterior.

En lo que respecta al aprendizaje competitivo con el que cuenta SOM, se trata de que las neuronas compitan unas con otras con la finalidad de desarrollar una tarea concreta y así especializarse. Se pretende que cuando se presente a la red un patrón de entrada, sólo una de las neuronas de salida (o un grupo de vecinas) se active. La neurona ganadora (C) se determina con la siguiente expresión:

$c=\arg \min _{i}\left(\left\|\mathbf{x}-W_{i}\right\|\right)$

Donde $\mathrm{x}$ es el vector de entrada y $\mathrm{W}_{\mathrm{i}}$ son los pesos de cada neurona del SOM.

Por tanto, las neuronas compiten por activarse, quedando finalmente una de ellas, como neurona vencedora, y anuladas el resto, que son forzadas a sus valores de respuesta mínimos.

El objetivo de este tipo de aprendizaje (competitivo), es categorizar los datos que se introducen en la red. Se clasifican valores similares en la misma categoría y, por tanto, deben activar la misma neurona de salida. Las clases o categorías deben ser creadas por la propia red a través de las correlaciones entre los datos de entrada, puesto que se trata de un aprendizaje no supervisado.

Un modelo SOM está compuesto por dos capas de neuronas:

- Capa de entrada: (formada por $\mathrm{N}$ neuronas, una por cada variable de entrada) se encarga de recibir y transmitir a la capa de salida la información procedente del exterior.

- Capa de salida: (formada por M neuronas) es la encargada de procesar la información y formar el mapa de rasgos. Normalmente, las neuronas de la capa de salida se organizan en forma de mapa bidimensional.

Las conexiones entre las dos capas que forman la red son siempre hacia delante, es decir, la información se propaga desde la capa de entrada hacia la capa de salida. Cada neurona de entrada i está conectada con cada una de las neuronas de salida $\mathrm{j}$ mediante un peso $\mathrm{W}_{\mathrm{ji}}$. De esta forma, las neuronas de salida tienen asociado un vector de pesos $W_{j}$ llamado vector de referencia (o codebook), debido a que constituye el vector prototipo (o promedio) de la categoría representada por la neurona de salida j. De esta manera, el SOM consigue detallar una proyección desde un espacio de datos en alta dimensión a un mapa bidimensional de neuronas.

Entre las neuronas de la capa de salida, puede decirse que existen conexiones laterales de excitación e inhibición implícitas, pues aunque no estén conectadas, cada una de estas neuronas va a tener cierta influencia sobre sus vecinas. Esto se consigue a través de un proceso de competición entre las neuronas y de la aplicación de una función denominada de vecindad, que produce la topología o estructura del mapa.

Las topologías más frecuentes son la rectangular y la hexagonal. El número de neuronas determina la suavidad de la proyección, lo cual influye en el ajuste y capacidad de generalización del SOM.

Los SOM han sido ampliamente utilizados, con multitud de trabajos que los aplican. En esta ocasión, son utilizados por primera vez en la investigación sobre las crisis económicas y la siniestralidad laboral.

\section{RESULTADOS}

El objetivo primordial de este estudio es averiguar si existe alguna relación entre los ciclos económicos y la siniestralidad laboral. El hecho de que uno y otro aspecto estuvieran ligados entre sí de algún modo, podría en un futuro, ayudar a prevenir posibles accidentes laborales, basándose en el comportamiento de los distintos indicadores macroeconómicos, 
evitando a su vez, importantes pérdidas humanas y económicas.

Con este estudio además se pretende poder dibujar un mapa descriptivo temporal (por años) del comportamiento de los ciclos económicos y otro de la siniestralidad; tratando de ver si ambos mapas guardan alguna relación, así como las variables que en ellos se representan.

\subsection{RESULTADOS - VARIABLES MACROE- CONÓMICAS}

Desde el momento en el que se inicia una crisis económica, los principales indicadores económicos presentan los primeros cambios referidos a la economía, siendo las variables macroeconómicas los principales indicadores a través de los que se puede medir la evolución económica de un país, por lo que es obligado estudiar su comportamiento a lo largo del periodo temporal seleccionado para este estudio.

Para realizar este estudio se van a aplicar técnicas inteligentes que permitan analizar el comportamiento de las variables macroeconómicas a lo largo del tiempo, con el objetivo de identificar las crisis económicas, las épocas de bonanza o crecimiento económico, y demás situaciones de la economía de nuestro país. Los fundamentos de estas técnicas han sido descritas en el apartado anterior relativo a las metodologías y los resultados de su aplicación. Los resultados se han ob- tenido con los datos tomados de las fuentes citadas anteriormente, los cuales se indican a continuación.

\subsubsection{Principal Component Analysis}

En las proyecciones obtenidas, los ejes verticales y horizontales que forman estas, son combinaciones de las diferentes variables originales. En estas visualizaciones (scatterplot), cada punto representa uno de los datos originales, representándose junto con el número del año correspondiente

En el caso de PCA, las nuevas dimensiones obtenidas son aquellas que maximizan la varianza en los datos (componentes principales), siendo cada una de ellas ortogonal a las demás y recogiendo un porcentaje de la varianza original de los datos.

En la aplicación del modelo, se ha fijado en tres el número de componentes principales a obtener, ya que estas tres son suficientes para explicar los datos de manera habitual. Esto se comprueba en el caso del estudio, ya que las tres primeras componentes tienen asociados respectivamente los siguientes coeficientes de varianza respecto de los datos originales: $77^{\prime} 5689 \%$, 11'4886\% y 4'5904\%. Por tanto, de las tres posibles combinaciones de componentes, sólo se muestran las combinaciones de los dos primeros, ya que explican el 89, 0575\% de la varianza de los datos.
Gráfico 3 Proyección PCA de las dos componentes principales (combinación 1-2) Variables macroeconómicas Fuente: Elaboración propia con datos del INE

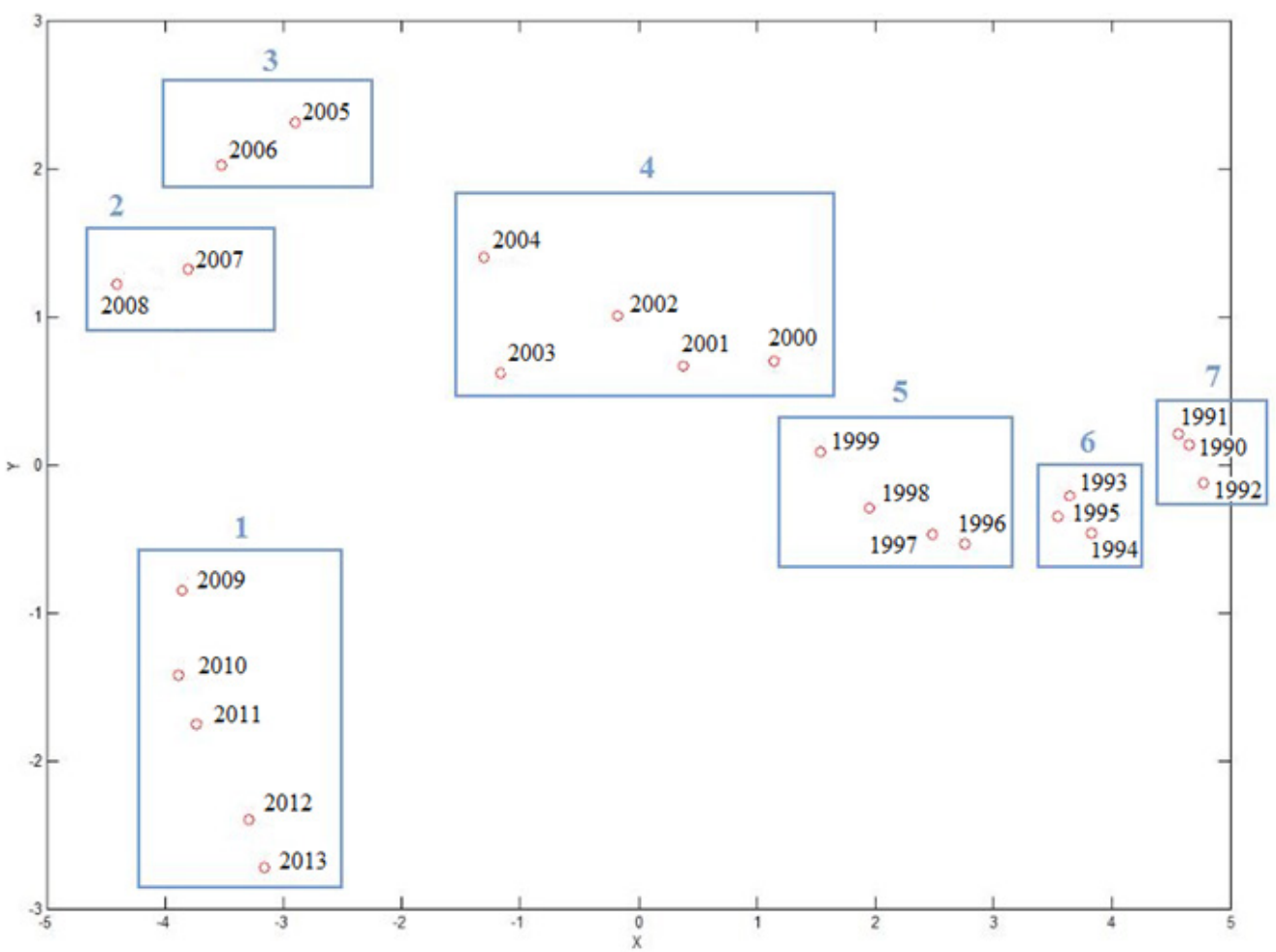


En el gráfico 3 se puede observar cómo los puntos asociados a los años bajo estudio se organizan con una cierta estructura. Para detallar y analizarlos más claramente, se ha decidido separar los puntos en distintas áreas, las cuales se corresponden con los ciclos económicos.

Con PCA y el análisis de sus dos primeras componentes principales (1-2), se obtiene una proyección que muestra un ordenamiento casi "lineal" de los datos, agrupando estos por áreas según los valores que van tomando las diferentes variables macroeconómicas a lo largo del tiempo. Gracias a la semejanza o similitud que se establece entre los distintos puntos (años), se puede observar cómo años en los que las condiciones económicas han sido similares, se agrupan juntos en el mismo área, y a su vez, dicho área se encuentra cerca de otro área de similares características, pero con matices que la diferencian de la anterior.

\subsubsection{Cooperative Maximum Likelihood Hebbian Learn- ing}

A continuación se han realizado numerosos entrenamientos del modelo neuronal Cooperative Maximum Likelihood
Hebbian Learning (CMLHL) con distintos valores para sus parámetros. De todos ellos se ha seleccionado sólo uno, el que se muestra en este apartado, debido a la convergencia alcanzada en el entrenamiento y la calidad del resultado.

En el gráfico 4 se muestra la proyección CMLHL en dos dimensiones del componente 1-2, el cual contiene escenarios infrecuentes sobre la situación económica nacional a lo largo del periodo de estudio. Esta se ha obtenido con los siguientes valores para los distintos parámetros:

Learning Rate: 0'040095 / p: 1'97 / Tau: 0'050008 / $\mathrm{N}^{\mathrm{o}}$ de iteraciones: 80

Se observa claramente en el gráfico 3 cómo el modelo es capaz de representar una estructura bien definida de los datos. Esto permite identificar 6 grandes áreas que han sido numeradas (del 1 al 6) y que se corresponden a su vez con las distintas fases de los ciclos económicos nacionales.
Gráfico 4 Proyección CMLHL (combinación 1-2) de las variables macroeconómicas

Fuente: Elaboración propia con datos del INE

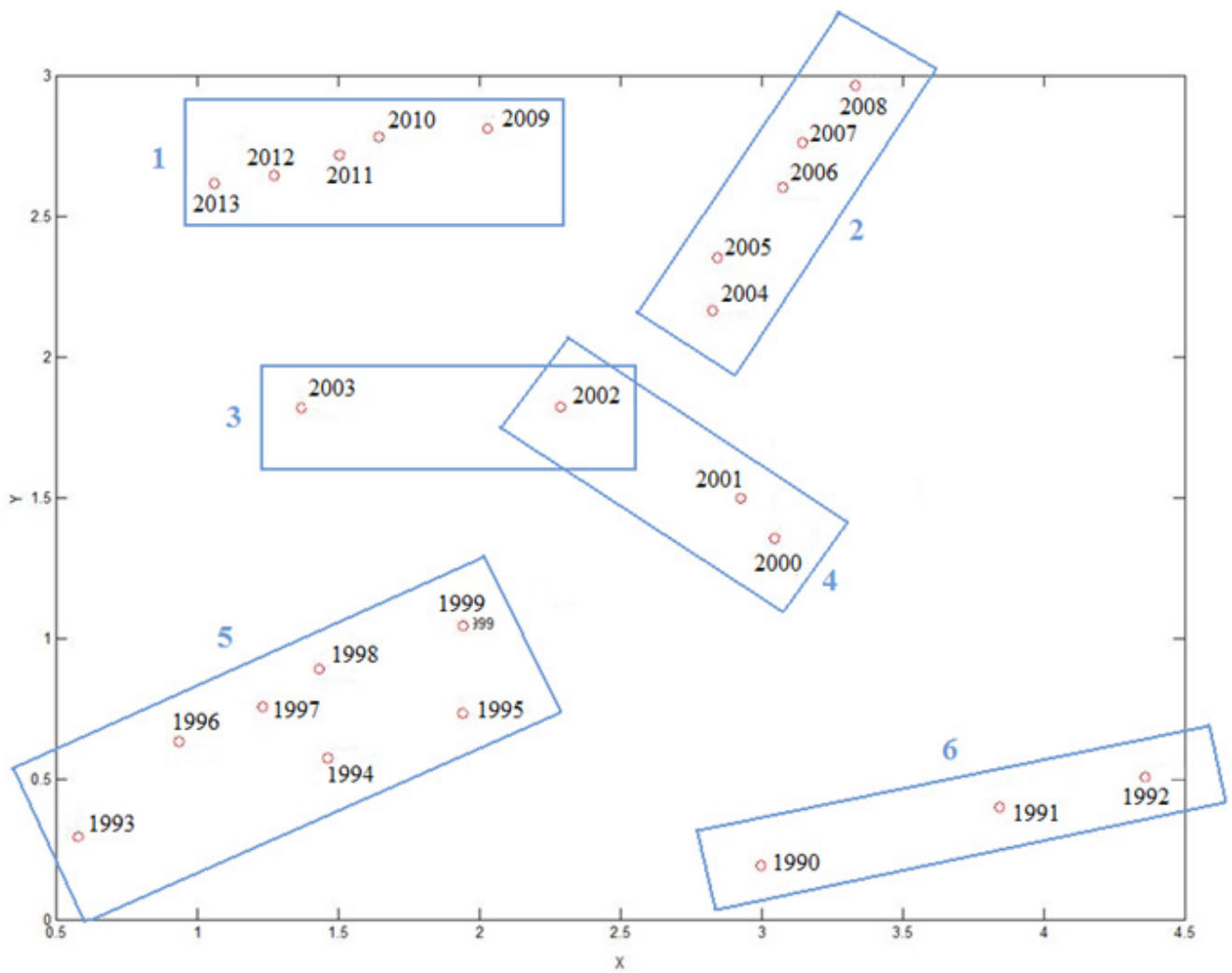


El hecho de que las distintas crisis se sitúen en áreas diferentes y separadas, e incluso, distantes entre sí, refleja la gran diferencia que existe entre ambas crisis a todos los niveles(alcance, causa-origen, duración, etc.). Algunos datos diferentes en la comparativa de ambas crisis son por ejemplo, el número de parados en la "crisis de los noventa", el cual aumenta en torno al 12\%, mientras que en la crisis actual lo hace un $41 \%$ en 2008 y un $60 \%$ en 2009 . La contracción del PIB de 1991 a 1992 es del 9\%, mientras que de 2008 a 2009 es del $3 \%$, etc.

Comparando las distintas proyecciones obtenidas para los datos macroeconómicos (PCA y CMLHL) se puede concluir que los resultados de CMLHL respaldan, afianzan y aclaran la estructura obtenida con PCA, permitiendo una más fácil identificación de los grupos y añadiendo más información al conjunto de resultados obtenidos de los experimentos realizados mediante PCA. Cabe destacar por tanto que agrupando los años similares (como se ha observado también para PCA), además en el caso de CMLHL se mejora la proyección obtenida al ajustarse ésta a la topología de los datos de entrada, preservando la cercanía entre grupos de datos similares y a la inversa. Este es precisamente el efecto que se persigue con la inclusión de conexiones laterales entre las neuronas de la capa de salida.

La proyección de PCA, y en especial la de CMLHL, permiten identificar escenarios anómalos, chocantes o infrecuentes, algunos de estos escenarios dependiendo del año del que se trate, tendrán una peculiaridad que los diferenciarán del resto de años o áreas. Esta singularidad puede asociarse a un año de bonanza económica, o a un conjunto de años de recesión, etc. La diferencia que presentan con el resto del conjunto de años y otras peculiaridades que se han visto reflejadas en la experimentación y en dicho análisis, reflejan escenarios infrecuentes, permitiéndose así, su fácil identificación mediante una intuitiva visualización de los datos macroeconómicos utilizados en el estudio.

\subsubsection{Self-Organizing Map}

Las redes neuronales artificiales del tipo Self Organizing Map (SOM) permiten obtener mapas de características que recogen las muestras de entrada. En el presente estudio las muestras de entrada son los diferentes años que se mapean a partir de un espacio multidimensional de entrada, en este caso formado por las variables macroeconómicas seleccionadas. Estos modelos se diferencian por tanto de los dos anteriormente aplicados (PCA y CMLHL) ya que no proporcionan una proyección de los datos sino una asignación de los mismos a las distintas neuronas incluidas en la rejilla de salida.

Se han realizado distintos entrenamientos probando con distintos valores para estos parámetros. A continuación (Gráficos 5 y 6) se muestra sólo uno de los resultados (SOM3 ), escogido entre una selección de los mejores resultados obtenidos. En él se reflejan dos elementos; por un lado la matriz de distancias, que permite visualizar cómo de cercanas se encuentran las neuronas de la rejilla y por otro, la propia rejilla de neuronas etiquetando cada neurona con los años para los que está respondiendo cada una de ellas.

Los resultados SOM-3 que se muestran, se corresponden con un entrenamiento de SOM sobre los datos macroeconómicos aplicando los siguientes valores:

Inicialización: Lineal / Algoritmo de entrenamiento: Secuencial / Tamaño del mapa: Grande / Rejilla: Hexagonal / Función de vecindad: Gaussiana

Como medida de la calidad del resultado generado, se recogen los valores para los dos principales indicadores en el caso de SOM-3:

Error de cuantización final: 0’641 /Error topográfico final: 0'042

En este experimento el tamaño del mapa es grande, lo cual nos permitirá encontrar más matices diferenciadores entre los distintos años bajo estudio. En el gráfico 5 se muestra la matriz de distancias y en el gráfico 6 la rejilla de neuronas para el experimento SOM-3. 
Gráfico 5 Matriz de distancias SOM-3 para variables macroeconómicas

Fuente: Elaboración propia con datos del INE
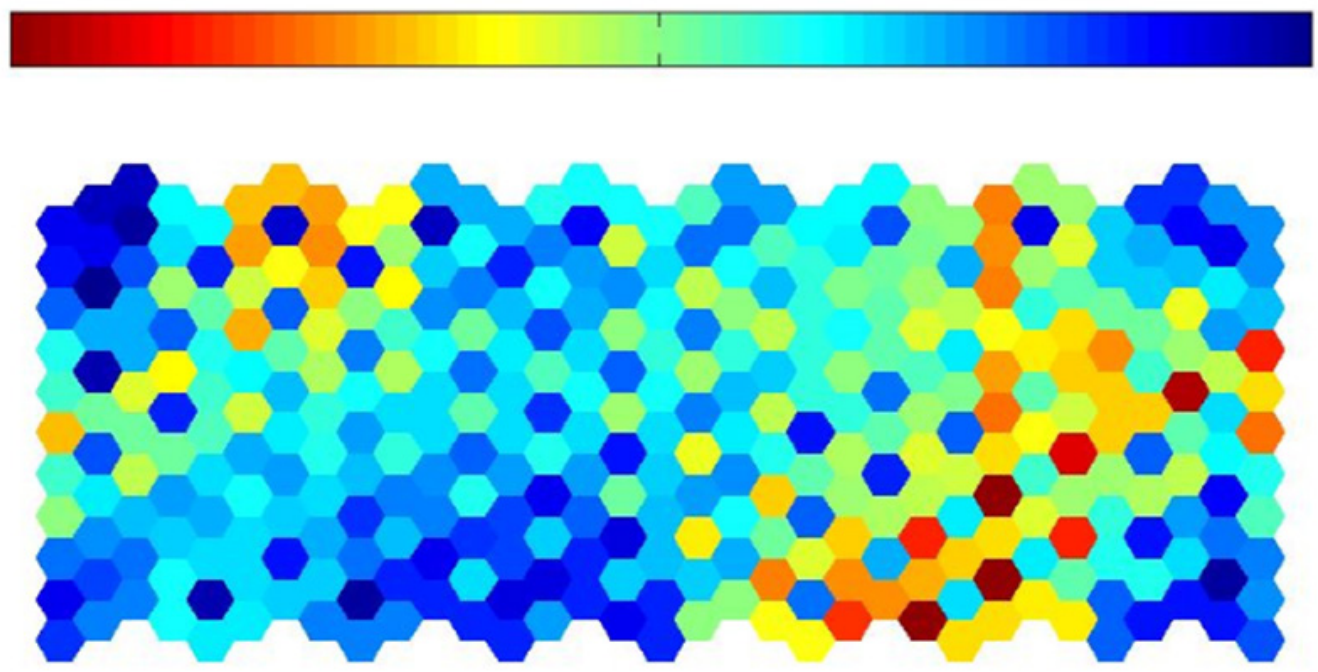

Gráfico 6 Rejilla de neuronas SOM-3 para variables macroeconómicas Fuente: Elaboración propia con datos del INE

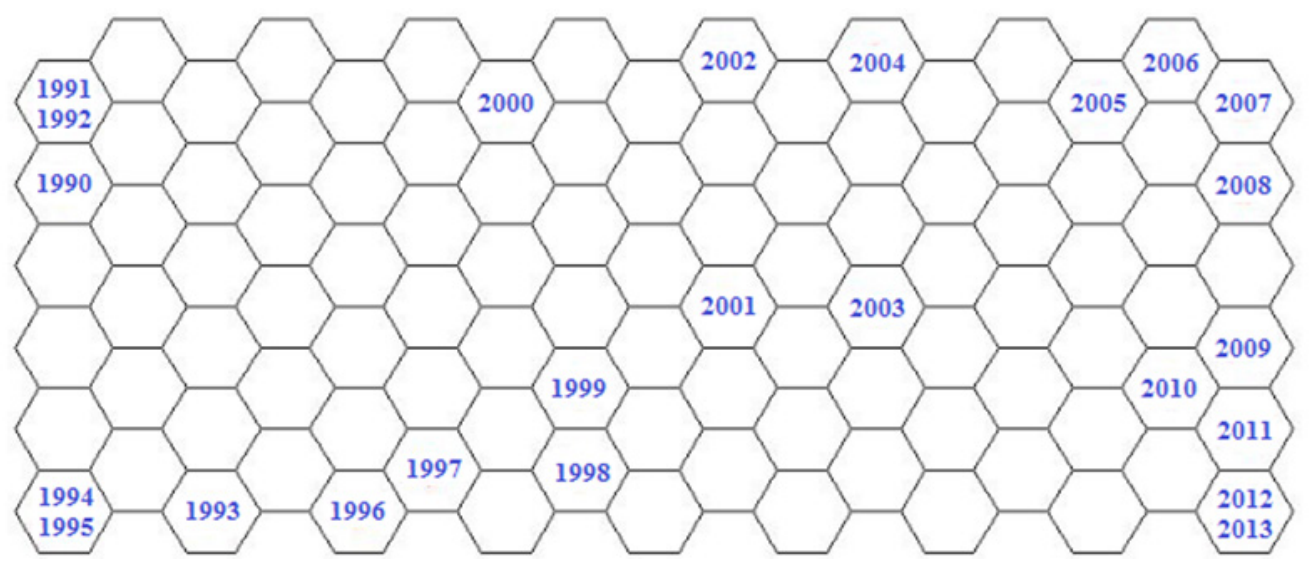

Se observa, tanto en la rejilla de neuronas (Gráfico 6) como en la matriz de distancias (Gráfico 5), que una gran distancia separa a las neuronas que comprenden ambas crisis, pues se encuentran en esquinas opuestas del mapa y entre ellas existe una gran separación; es decir, que aunque ambos periodos han sido de recesión económica, realmente, no se han desarrollado y manifestado del mismo modo.

\subsection{RESULTADOS - VARIABLES DE SINI- ESTRALIDAD}

Las variables de siniestralidad nos permiten cuantificar y medir la accidentalidad laboral a lo largo del tiempo. En el caso de las variables de siniestralidad es aún más difícil disponer de datos completos para grandes series de años ya que a lo largo del tiempo el gobierno ha ido cambiando la configuración de las distintas variables, con lo cual, para el presente estudio se han tenido que desestimar algunas de ellas, por no disponer de datos uniformes en la totalidad del periodo analizado.

\subsubsection{Principal Component Analysis}

En el Análisis de Componentes Principales (PCA) aplicado a las variables de siniestralidad, el número de componentes principales a obtener, para la aplicación del modelo, se ha fijado en 3, puesto que estas son suficientes para explicar en gran medida los datos. Se comprueba en el caso del estudio que las 3 primeras componentes tienen asociados respectivamente los siguientes coeficientes de varianza respecto de los datos originales: $59^{\prime} 8868 \%$, 33'0985\% y 3'6133\%. En la proyección siguiente (Gráfico 7), que muestra la combinación de componentes principales 1-2, se explican la mayor parte de varianza de los datos, que asciende al 92'9853\%. 
Gráfico 7 Proyección PCA de las dos componentes principales (combinación 1-2 del Gráfico V.S.1)

Variables de siniestralidad

Fuente: Elaboración propia con datos del INE

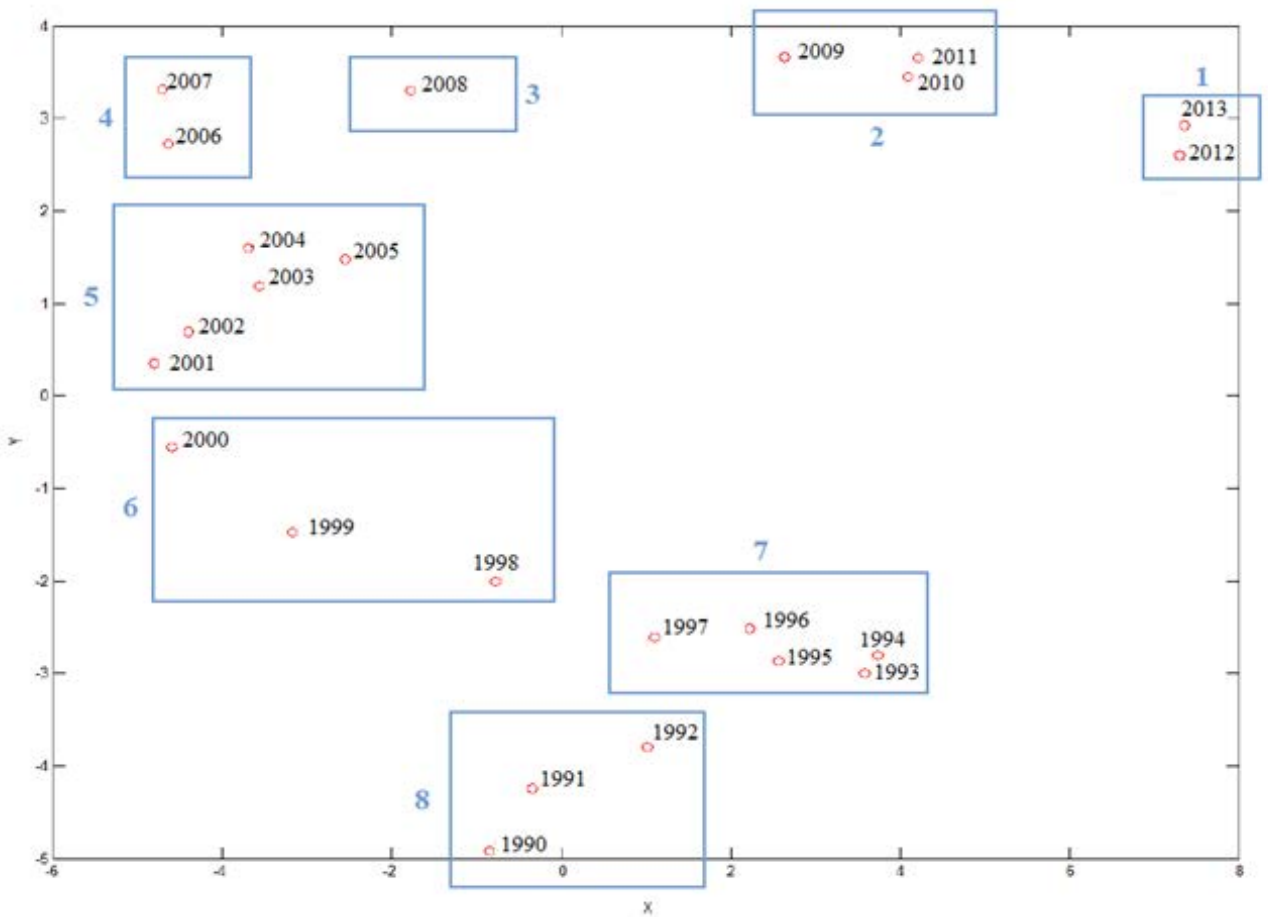

El agrupamiento de estos años según las distintas áreas marcadas en el gráfico 6 , permiten diferenciar con claridad cada una de las fases por las que pasa la siniestralidad laboral a lo largo del periodo seleccionado, distinguiéndose entre periodos de crecimiento o descenso de la misma.

\subsubsection{Cooperative Maximum Likelihood Hebbian Learn- ing}

Partiendo de las variables de siniestralidad anuales se ha aplicado también el modelo CMLHL sobre los datos del periodo. En el gráfico 8 se muestra la scatterplot matrix asociada a las proyecciones obtenidas a partir de dichas variables. Se han realizado numerosos entrenamientos del modelo neuronal CMLHL con diferentes valores para sus parámetros; de todos ellos, solo se ha seleccionado el que proporciona una mejor convergencia en el entrenamiento y ofrece una mejor calidad de resultados.

La proyección CMLHL del gráfico 7 se ha obtenido con los siguientes valores para los distintos parámetros:

Learning Rate: 0'12009 / p: 1'75 / Tau: 0'14 / No de iteraciones: 60 
Gráfico 8 Proyección CMLHL (combinación 1-2 del Gráfico III.S.3) de las variables de siniestralidad Fuente: Elaboración propia con datos del INE

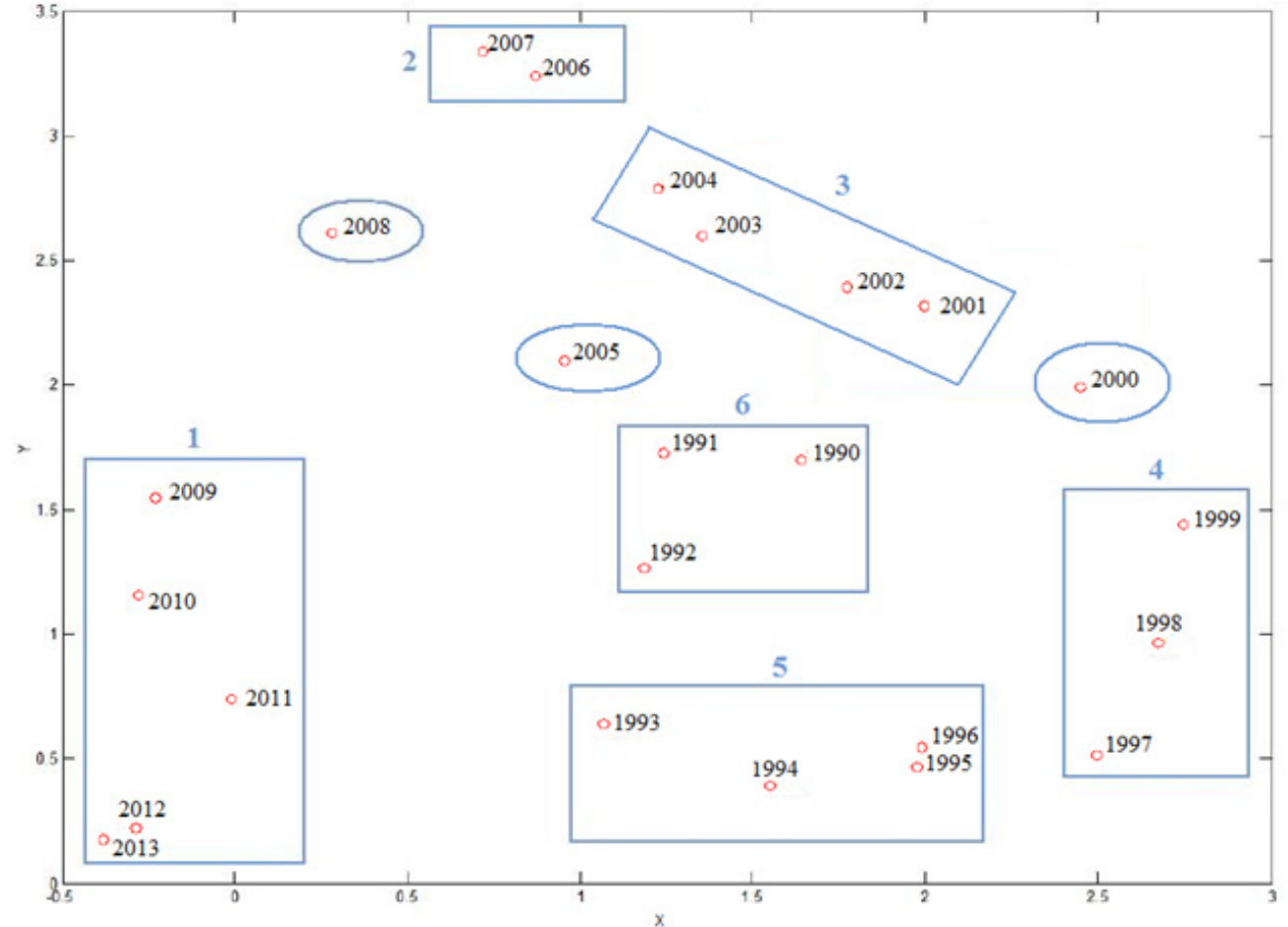

Comparando las proyecciones (PCA y CMLHL) obtenidas para los datos de siniestralidad, se considera que nuevamente los resultados de CMLHL aclaran y enriquecen la estructura evidenciada por PCA. Esto permite una más fácil identificación de los grupos y añade más información al conjunto de resultados obtenidos respecto a los experimentos realizados con PCA.

\subsubsection{Self-Organizing Map}

Como en el caso anterior, se han desarrollado distintos entrenamientos con este modelo, probando con distintos valores para los parámetros que éste emplea. A continuación se recoge únicamente el mejor resultado obtenido.

Los resultados SOM-2 que se muestran, se corresponden con el experimento SOM, sobre los datos de siniestralidad del periodo analizado, aplicando los siguientes valores:
Inicialización: Lineal / Algoritmo de entrenamiento: Secuencial / Tamaño del mapa: Grande / Rejilla: Hexagonal / Función de vecindad: Gaussiana

Como medida de la calidad del resultado generado, se recogen los valores para los dos principales indicadores en el caso de SOM-2:

Error de cuantifización final: 0'681 / Error topográfico final: $0{ }^{\prime} 000$

Observando simultáneamente la matriz de distancias (Gráfico 9) y la rejilla de neuronas del entrenamiento SOM2 (Gráfico 10), se aprecia la existencia de cuatro zonas de neuronas, separadas entre sí por grandes distancias. En estos gráficos se observa cómo las grandes dimensiones de la rejilla hacen que los datos se encuentren menos concentrados y que de esa manera se produzca una mayor especialización de las neuronas. 
Sonia Contreras Ovejero et al. / Dirección y Organización 65 (2018) 5-19

Gráfico 9 Matriz de distancias SOM-2 para variables de siniestralidad

Fuente: Elaboración propia con datos del INE.

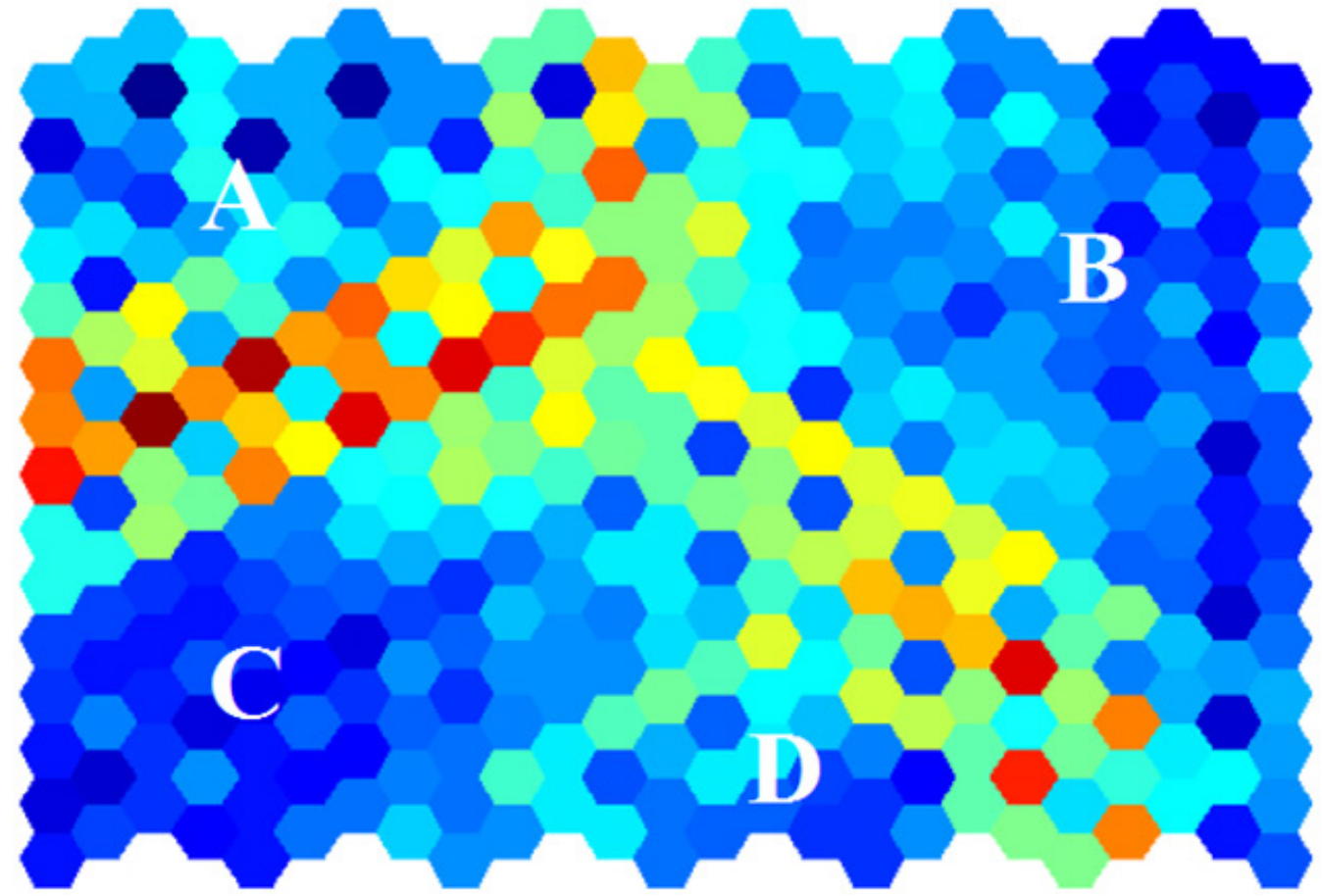

Gráfico 10 Rejilla de neuronas SOM-2 para variables de siniestralidad

Fuente: Elaboración propia con datos del INE

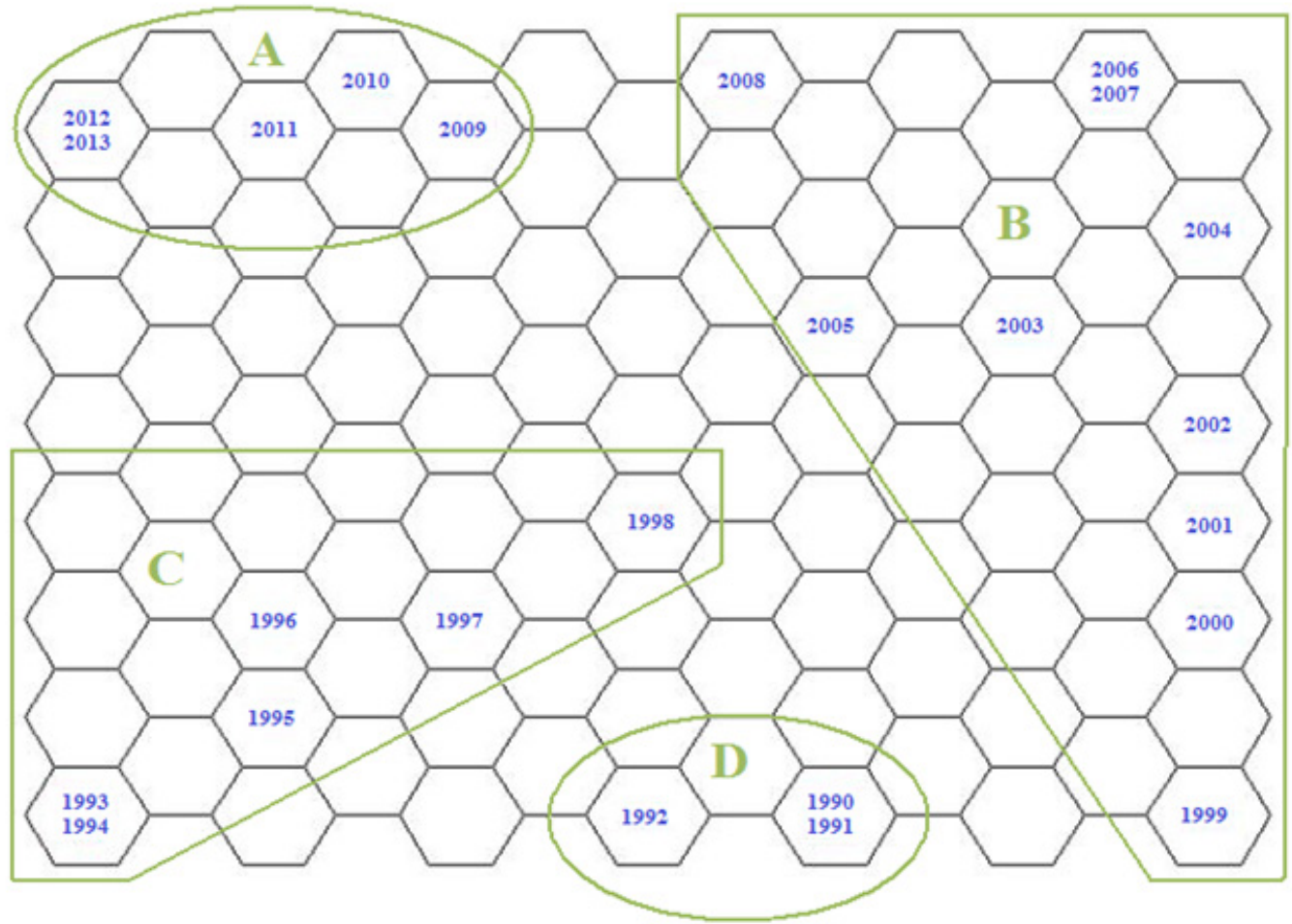


Las cuatro zonas en las que se distribuyen los años en el mapa obtenido en el experimento SOM-2 también guardan gran correspondencia y similitud con los ciclos económicos acontecidos en el mismo periodo temporal. Este hecho parece reforzar la existencia de un nexo de unión entre la siniestralidad laboral y los ciclos económicos.

Tras el análisis de las variables de siniestralidad, se puede destacar que la visualización de estos datos a través de los modelos neuronales es coherente con la de los datos macroeconómicos, lo cual apunta a que puede existir relación entre ambos (variables macroeconómicas y de siniestralidad laboral)

\section{CONCLUSIONES}

El estudio planteado trata de dar continuidad a trabajos realizados anteriormente sobre siniestralidad laboral y ciclo económico en España, como (Martín-Román ,2006) y (Arango y Valdivia, 2000), entre otros, en los que se constata la estrecha relación proporcional directa entre el aumento de la siniestralidad laboral y el crecimiento económico. En estos trabajos no se contempla su relación en los periodos de crisis, por lo que se ha considerado interesante y necesario estudiar en profundidad lo que ocurre específicamente durante estos períodos.

El hecho de considerar las crisis económicas como una variable más de siniestralidad, cobra sentido a lo largo de todo este estudio, puesto que en múltiples ocasiones, y a través de distintos métodos, se ha podido comprobar cómo ambas se encuentran estrechamente relacionadas entre sí. La accidentalidad depende en gran medida de la situación económica nacional, ya que como se ha observado, la siniestralidad se reduce en tiempos de crisis, y aumenta en épocas de bonanza económica.

Los resultados obtenidos a través de los modelos neuronales, aplicados a ambos tipos de variables, permiten apreciar con claridad una distribución anual muy análoga entre sí. Se puede apreciar cómo en los años de crisis se agrupan entre sí, así como también ocurre en los años de crecimiento económico. Queda por lo tanto plasmado en las visualizaciones neuronales la evidencia de la relación que existe entre ambos tipos de variables, ya que dicha distribución es prácticamente la misma en todos los casos

Los dos conjuntos de variables analizados muestran una estructura de datos anuales muy similar; y estos resultados no pueden ser fruto de la coincidencia, ya que se han comparado distintas técnicas, se han seleccionado distintas variables y años; obteniendo en todas ellas resultados muy similares entre sí.
Por todo ello se puede afirmar que entre las crisis económicas y la siniestralidad existe una estrecha relación, y que la accidentalidad varía en función de los ciclos económicos, de ahí esa relación de dependencia por parte de la siniestralidad hacia las crisis económicas y los ciclos económicos.

\section{BIBLIOGRAFÍA}

1. Alcázar, P. (2014). "El día después de la crisis: qué legado nos dejan siete años de duros ajustes en los principales indicadores macroeconómicos”. Emprendedores. Las claves de la economía y el éxito profesional. no.198, p.18-22.

2. Alonso-Nuez, MJ; Flores-García, M; Muñoz-Porcar, A. (2015) "Construction industry in Spain, is it guilty of the current crisis?". Applied Economics. vol.47, no.37, p.3994-4006.

3. Arango, J; Valdivia, E. (2000). "Siniestralidad laboral y crecimiento económico en España”. Revista Asturiana de Economía. vol.1, no.18 p.47-71.

4. Audiffren, AT; Guarnieri, F; Martin, C; et al. (2014). "A quantitative analysis of health, safety and environment policy in France". Safety, Reliability and Risk Analysis: Beyond the Horizon. vol.1, p.1639-1643.

5. Banco de España (BDE). www.bde.es

6. Bertsekas, DP. (1999). "Nonlinear Programming. Athena Scientific”. Belmont, MA.

7. Bishop, CM. (1995). "Neural Networks for Pattern Recognition”. Oxford.

8. Castella, JL. (2000). "Accidentes, empleo, carga de trabajo y peligrosidad del trabajo”. Prevención, Trabajo y Salud: Revista del Instituto Nacional de Seguridad e Higiene en el Trabajo. ISSN.1575-1392, p.16-26.

9. Corchado, E; Fyfe, C. (2003). "Connectionist techniques for the identification and suppression of interfering underlying factors". International Journal of Pattern Recognition and Artificial Intelligence. vol.17, no.8, p.1447-1466.

10. Corchado, E; MacDonald, D; Fyfe, C. (2004). "Maximum and Minimum Likelihood Hebbian Learning for Exploratory Projection Pursuit”. Data Mining and Knowledge Discovery. vol.8, no.3, p.203-225.

11. EUROSTAT. http://ec.europa.eu/eurostat. Elaboración de estadísticas. Último acceso: 25-Octubre-2015. 
12. Friedman, JH; Tukey, JW. 1974. "A projection pursuit algorithm for exploratory data análisis”. IEEE Transact. Computers. vol.23, p.881-889.

13. García, I; Montuenga, V. (2009). "Causas de los accidentes de trabajo en España: análisis longitudinal con datos de panel". Gaceta Sanitaria. vol.23, no.3, p.174178 .

14. Herrero, A; Corchado, E; Sáiz, J.M. (2006). "MOVICAB-IDS: visual analysis of network traffic data streams for intrusion detection”. Intelligent Data Engineering and Automated Learning - IDEAL 2006. Springer, Lecture Notes in Computer Science, vol. 4224, p. 1424-1433.

15. Herrero, A; Corchado, E; Saiz, L; Abraham, A. (2010). "DIPKIP: A connectionist knowledge management system to identify knowledge deficits in practical cases". Computational Intelligence. vol.26, no.1, p.26-56.

16. Herrero, A; Corchado, E; Jiménez, A. (2011). "Unsupervised neural models for country and political risk analysis”. Expert Syst. Appl. vol.38, no.11, p.13641-13661.

17. Hotelling, H. (1933) "Analysis of a complex of statistical variables into principal components”. Journal of Education Psychology. vol.24, p.417-444.

18. Huang, S; Yang, D; Ge Y; et al. (2015). "Combined supervised information with PCA via discriminative component selection”. Information Processing Letters”. vol.115, no.11, p.812-816.

19. Instituto Nacional de Estadística del Gobierno de España (INE). www.ine.es

20. Instituto Nacional de Seguridad e Higiene en el Trabajo (INSHT). www.insht.es

21. Jimeno, JF; Santos, T. (2014). "The crisis of the Spanish Economy". Series-Journal of the Spanish Economic Association". Vol.5 N.2-3 p-p. 125-141. Franco A. "Enfoque para el análisis de la crisis económica global y la salud”. Revista Facultad Nacional de Salud Pública. vol.27, no.3, p.329-340.

22. Kohonen, T. (1982). "Self-organized formation of topologically correct feature maps”. Biological Cybernetics. vol.43, no.1, p.59-69.
23. Liu, Z; Chai, T; Yu, W; et al. (2014). "Multi-frequency signal modeling using empirical mode decomposition and PCA with application to mill load estimation”. Conferencia: 11th World Congress on Intelligent Control and Automation (WCICA). Shenyang. Jun.27-30. Neurocomputing. vol.169, p.392-402.

24. Lozano, L. (1999). "Estudio Bio-ecológico de los vectores de la enfermedad de Chagas en el Municipio de Coyaima - Tolima”. Tesis de grado en Biología. Universidad del Tolima Ibagué.

25. Manzanedo, MA; Sáiz Bárcena, L. (1996). “Organización de la Seguridad Industrial”. Editorial: Centro Tecnológico Industrial, Burgos. ISBN:84-921656-O-X.

26. Martín-Román, A. (2006). "Siniestralidad laboral y ciclo económico: ¿Una relación meramente estadística o un fenómeno real?”. Revista del Ministerio de Trabajo y Asuntos Sociales. vol.61, p.157-171.

27. Ministerio de Empleo y Seguridad Social del Gobierno de España (MEYSS). www.meyss.es

28. Moënne, K. (2008). “La crisis financiera y la salud”. Revista Chilena de Radiología. vol.14, no.4, p.179-179.

29. Organización Internacional del Trabajo. (OIT). http:// www.ilo.org/global/lang--es/index.htm. Último acceso: 25-Octubre-2015.

30. Papazoglou, IA; Aneziris, O; Bellamy, L; et al. (2015). "Uncertainty Assessment in the Quantification of Risk Rates of Occupational Accidents". Risk Analysis. vol.35, no.8, p.1536-1561.

31. Pearson, K. (1901) "On Lines and Planes of Closest Fit to Systems of Points in Space”. Philosophical Magazine. vol.2, no.6, p.559-572.

32. Sánchez, R; Herrero, A; Corchado, E. (2013). "Visualization and Clustering for SNMP Intrusion Detection". Cybernetics and Systems. vol.44, no.6-7, p.505-532.

33. Seung, HS; Socci, ND; Lee, D. (1998). "The Rectified Gaussian distribution”. Advances in Neural Information Processing Systems. vol.10, p.350-356.

34. Teixidó, P. (2009). "La prevención en época de crisis". Prevención: Revista técnica de seguridad y salud laborales. no.189, p.20-33. 
35. Vázquez, G. (2007). "La crisis del Sistema Monetario Europeo (1992-1993): ¿crisis financiera o crisis de políticas de cooperación monetaria?”. Departamento de Economía Aplicada I. Universidad Complutense de Madrid.

36. Villanueva, V; Clemente, I. (2001). "Accidentes de trabajo y factores económicos asociados”. Archivos Prevención de Riesgos Laborales. vol.4-1, p.6-15.
37. Wang, T; Xu, H; Han, J; et al. (2015). "Cascaded H-Bridge Multilevel Inverter System Fault Diagnosis Using a PCA and Multiclass Relevance Vector Machine Approach”. IEEE Transactions on Power Electronics. vol.30, no.12, p.7006-7018. 